\title{
Against non-reference-tracking theories of switch-reference
}

\author{
Emily Clem*
}

\begin{abstract}
Recent accounts of switch-reference have suggested that direct reference tracking is not involved. Instead, these accounts have sought to derive patterns of switch-reference from other independently attested phenomena such as control and coordination. What these diverse theories have in common is the prediction that same subject constructions should contain only one instance of a subject DP. I present evidence from Amahuaca showing that overt DP subjects can appear in both clauses in same subject constructions, contra the predictions of these nonreference-tracking theories. However, there is also evidence that Amahuaca same subject marked clauses are structurally smaller than different subject marked clauses. This size asymmetry is predicted by non-reference-tracking accounts but not by traditional direct reference-tracking theories. Thus while the Amahuaca data provide evidence against non-reference-tracking theories of switch-reference, they suggest that direct reference-tracking accounts must also be modified in order to account for the full range of data.
\end{abstract}

Keywords. switch-reference; Amahuaca; same subject; control; coordination

1. Introduction. The term "switch-reference" (SR) was coined by Jacobsen (1967) to describe the phenomenon of obligatory morphological encoding of a change in subject. Early generative accounts of SR, such as Finer $(1984,1985)$, assumed that these constructions involved direct tracking of the referents of subjects, with coreferential subjects triggering same subject (SS) marking and disjoint subjects triggering different subject (DS) marking. However, some recent accounts of SR have diverged from their predecessors, assuming that SR is just a special instance of some other independently attested and more crosslinguistically widespread phenomenon such as control (Georgi, 2012) or coordination (Keine, 2012, 2013). While these non-referencetracking accounts differ significantly, what they have in common is the prediction that SS marking arises when there is only one subject DP shared between two predicates while DS marking arises when there are two distinct subject DPs in the structure.

In this paper, I present data from the Panoan language Amahuaca, in which SS constructions can contain an instance of a subject DP in both the marked and reference clause. ${ }^{1}$ The fact that SS constructions can contain two instances of a subject DP is evidence that nonreference-tracking accounts, such as those put forth by Georgi (2012) and Keine (2012, 2013), cannot be an adequate analysis of Amahuaca's SR system. However, I also demonstrate that there is a size asymmetry between SS and DS marked clauses in Amahuaca that is predicted by non-reference-tracking theories but not by traditional accounts of SR that appeal to direct reference tracking mediated by $\mathrm{C}$. Thus, while the Amahuaca data provide evidence against

${ }^{*}$ I would like to thank the members of the Amahuaca community for their collaboration. I am also grateful to Amy Rose Deal, Line Mikkelsen, Peter Jenks, and Dasha Kavitskaya for their input on this project. This work was made possible by 2015, 2016, and 2017 Oswalt Endangered Language Grants. All errors are mine alone. Author: Emily Clem, University of California, Berkeley (eclem@berkeley.edu).

${ }^{1}$ I follow (Munro, 1979) in using the term "marked clause" to refer to the clause that hosts the SR marker and "reference clause" to refer to the clause that hosts the other argument in the (non-)coreference relationship. 
non-reference-tracking theories, they also suggest that direct reference-tracking theories must be modified in order to account for the full range of data.

The structure of the remainder of the paper is as follows. In Section 2 I introduce some basic properties of Amahuaca's SR system. In Section 3 I introduce the accounts of SR proposed by Georgi (2012) and Keine (2012, 2013). I offer evidence against these theories based on the distribution of subject DPs in Amahuaca SS constructions in Section 4, but in Section 5 I demonstrate that these theories correctly predict the size asymmetry found in Amahuaca marked clauses. Finally, in Section 6 I offer concluding remarks.

2. Switch-reference in Amahuaca. Amahuaca is spoken in Peru and Brazil by approximately 500 speakers (Simons \& Fennig, 2017). Data presented here come from my fieldwork carried out between 2015 and 2017 with 11 native speakers of the language ( 8 female) in the district of Sepahua in Peru. Like other Panoan languages, Amahuaca has a rich SR system (SparingChávez, 1998, 2012). ${ }^{2}$ A basic example of an SS versus DS contrast is shown in (1). ${ }^{3}$
a. $\quad[\mathrm{jato}=\mathrm{x} \quad \mathrm{vua}=\mathrm{hi}]=$ mun $\quad$ chirin $=\mathrm{hi} \quad \mathrm{kan}=\mathrm{ki}=\mathrm{nu}$ $3 \mathrm{PL}=\mathrm{NOM}$ sing $=\mathrm{SS} . \mathrm{SIM} . \mathrm{NOM}=\mathrm{C}$ dance $=\mathrm{IPFV}$ 3PL $=3 . \mathrm{PRES}=\mathrm{DECL}$
'While they ${ }_{i}$ sing, they ${ }_{i}$ dance.'
b. $\quad[\mathrm{vaku}=\mathrm{vo}$ vua $=$ hain $]=$ mun chirin=hi $\mathrm{kan}=\mathrm{ki}=\mathrm{nu}$
child $=\mathrm{PL}$ sing $=\mathrm{DS} . \mathrm{SIM}=\mathrm{C}$ dance $=\mathrm{IPFV} 3 \mathrm{PL}=3 . \mathrm{PRES}=\mathrm{DECL}$
'While the children ${ }_{i}$ sing, they ${ }_{j}$ dance.'

In (1a) the subject of the marked clause is coreferential with the subject of the reference clause and the SS marker $=h i$ is used. In the minimally different (1b), the subject of the marked clause vakuvo 'children' is disjoint from the third person plural subject of the reference clause. In this case, the different subject marker $=$ hain is used.

In addition to encoding reference relationships between nominals, the SR markers of Amahuaca also encode temporal relationships between clauses, as seen in (2).
a. $\quad[$ hiya $=\mathrm{n}$ hun hano jiri $=$ kun $]=$ mun rato choka $=\mathrm{kan}=\mathrm{xo}=\mathrm{nu}$
$1 \mathrm{SG}=\mathrm{ERG} 1 \mathrm{SG}$ paca eat=DS.SQ=C plate wash $=3 \mathrm{PL}=3 . \mathrm{PST}=\mathrm{DECL}$

'After I ate paca, they washed plates.'

b. $\quad[$ hiya $=\mathrm{x}$ hun jiri $=$ hain $]=$ mun rato choka=hi $\mathrm{kan}=\mathrm{ki}=\mathrm{nu}$

$1 \mathrm{SG}=\mathrm{NOM} 1 \mathrm{SG}$ eat=DS.SIM=C plate $\mathrm{wash}=\mathrm{IPFV} 3 \mathrm{PL}=3 . \mathrm{PRES}=\mathrm{DECL}$

'While I eat, they are washing plates.'

In (2a), the event of the reference clause, washing plates, temporally follows the event of the marked clause, eating. This sequential action is indicated by the DS marker $=k u n$. In (2b) the plate-washing and eating are simultaneous, and the DS marker =hain is used instead.

Finally, the form of the SR marker in Amahuaca differs based on the grammatical function of the coreferential reference clause argument. SS markers are sensitive to a distinction

\footnotetext{
${ }^{2}$ Note that Sparing-Chávez uses the term "interclausal reference" to refer to the SR system of Amahuaca.

${ }^{3}$ The following abbreviations are used in glossing throughout: $1=$ first person, $3=$ third person, $\mathrm{C}=$ complementizer, $\mathrm{DECL}=$ declarative, $\mathrm{DS}=$ different subject, $\mathrm{ERG}=$ ergative, $\mathrm{IPFV}=$ imperfective, $\mathrm{NOM}=$ nominative, $\mathrm{PL}=$ plural, PRES = present, PST = past, $\mathrm{SG}=$ singular, $\mathrm{SIM}=$ simultaneous action, $\mathrm{SO}=$ subject coreferential with object, $\mathrm{SQ}=$ sequential action, $\mathrm{SS}=$ same subject.
} 
between transitive subjects versus intransitive subjects. Additionally, there is a marker which indicates that the subject of the marked clause is coreferential with the object of the reference clause. This three-way contrast is demonstrated in (3).
a. $[$ hoxa $=$ hax $]=$ mun xano vua $=x o=n u$
sleep $=$ SS.SQ.NOM $=C$ woman sing $=3$.PST $=$ DECL
'After sleeping, the woman sang.'
b. $[$ hoxa $=$ xOn $]=$ mun hiya xano=n $\quad$ vuna $=\mathrm{xO}=$ nu
sleep $=$ SS.SQ.ERG $=\mathrm{C}$ 1SG woman=ERG look.for=3.PST=DECL
After sleeping, the woman looked for me.'
c. [hatapa natuz $=\mathrm{XO}]=\mathrm{mun}$ joni=n hino hachi $=\mathrm{xO}=\mathrm{nu}$
chicken bite $=\mathrm{SO} . \mathrm{SQ}=\mathrm{C} \quad \mathrm{man}=\mathrm{ERG} \operatorname{dog}$ grab=3.PST=DECL
'After it bit the chicken, the man grabbed the dog.'

In (3a), there is coreference between the subject of the marked clause and the subject of the reference clause. The subject of the reference clause xano 'woman' is the subject of the intransitive verb vua 'sing', so the SS marker =hax appears. The example in (3b) is minimally different in that 'woman' is now the subject of the transitive verb vuna 'look for' in the reference clause. Instead of =hax, the SS marker =xon appears, indicating that the coreferential reference clause argument is a transitive subject. Finally, in (3c), the subject of the marked clause is coreferential with the object of the reference clause hino 'dog'. The SR marker $=x o$ indicates this subject-object coreference relationship. While the phenomenon of tracking transitivity of the reference clause is common in Panoan languages (Valenzuela, 2003; van Gijn, 2016), object tracking via SR is very rare crosslinguistically.

With this basic overview of the properties of Amahuaca's SR system in mind, we now turn to an overview of some recent analyses of SR.

3. Non-reference-tracking theories of SR. It has long been assumed that SR is about reference - as the name suggests. This insight has been the foundation of generative theories of SR such as those proposed by Finer $(1984,1985)$ and Watanabe (2000). Under these accounts, SR markers reflect whether arguments with the same referential index are present in two separate clauses. SR is thus taken to be related to Binding Theory in that the morphological realization of an element differs depending on whether another element with the same index stands in some special relation to it. SS marking is taken to be the analogue of an anaphor in the domain of SR, and DS marking is the SR analogue of a pronominal.

Some recent accounts of SR have departed from the traditional wisdom that SR tracks reference, and have instead sought to derive patterns of SR marking from other independently attested phenomena, such as control or coordination. While differing dramatically in the details of the proposals, what these accounts that eschew direct reference tracking have in common is the idea that SS constructions contain only one instance of a subject DP, while DS constructions contain two independent subjects. In Section 4, I will argue that such a prediction conflicts with the attested Amahuaca data. However, it is first useful to consider the details of these proposals.

The first non-reference-tracking account that I will consider is one proposed by Georgi (2012). Under this account, SS marked clauses are obligatory control structures, while DS marked clauses are non-control embedded clauses. Georgi analyzes SS clauses as TPs headed 
by a defective $\mathrm{T}$, which cannot assign case to the subject, leaving the subject DP active in the derivation. Adopting the movement theory of control following Boeckx et al. (2010), she argues that the active subject of the embedded TP moves into the matrix clause to satisfy the selectional features of the superordinate $v$. In the upstairs clause, this DP is assigned case. For Georgi, the SS marker is the spell out of the embedded defective T and indicates that T does not c-command any accessible DP since the subject was moved into a higher clause. The structure for an SS clause under this account is given in (4).

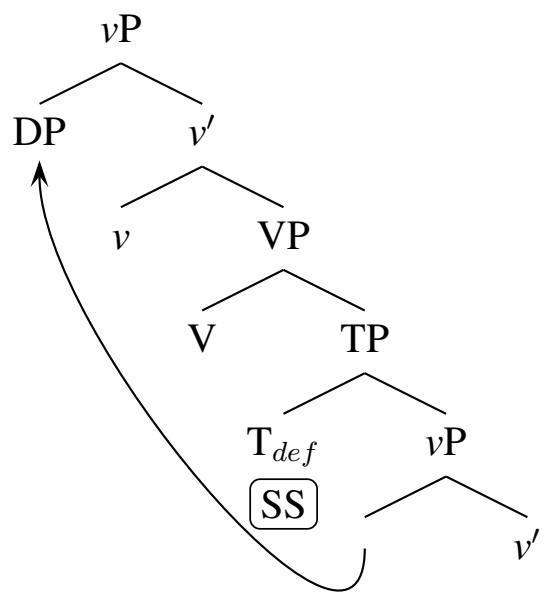

As can be seen in (4), the subject of the embedded TP moves into the higher clause, and the defective $\mathrm{T}$ of the embedded clause is spelled out as the SS marker.

Under Georgi's account, DS clauses are simply standard embedded CPs. The T of the embedded clause is able to case-mark the embedded subject, and a distinct DP is merged as the external argument of the higher clause. This structure is shown in (5).

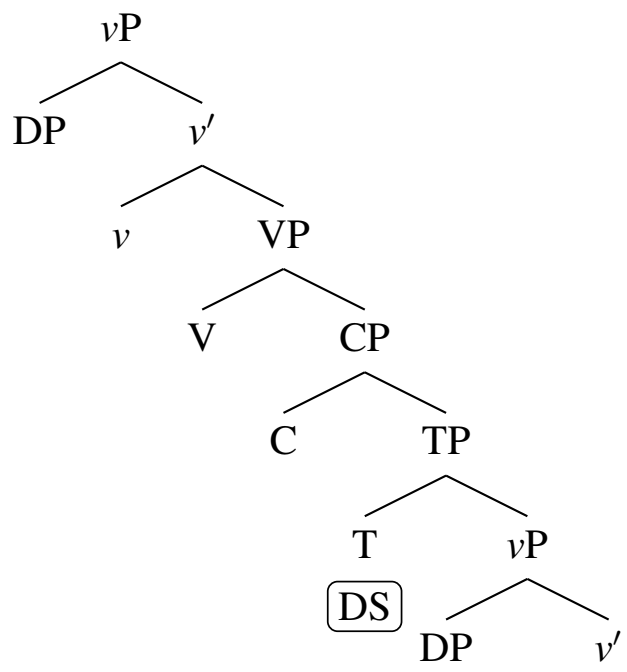

The structure in (5) involves an embedded CP. Note that both the main and embedded clause have a DP filling Spec, $v \mathrm{P}$ - the subjects of the two clauses are distinct.

There are a couple of important facets of this theory to keep in mind. The first is that SS structures should only have one instance of a subject DP. Each clause may potentially have a separate object, but the same DP that is merged as the subject of the embedded clause is moved to fill the subject position in the higher clause. The second important aspect of this 
account is that it predicts that there may be signs of the size asymmetry between SS and DS clauses. SS clauses are only TPs and are headed by a defective T, while DS clauses are full CPs with a regular T. Georgi points out that there does seem to be a size asymmetry between SS and DS clauses in many languages, with DS clauses often showing inflection and agreement that SS clauses lack.

The second non-reference-tracking theory of SR that I will consider here is that of Keine (2012, 2013). Keine assumes that SR structures involve coordination, and attributes the difference between SS and DS structures to the height of coordination. For Keine, SS clauses involve low coordination at the VP level. The SS marker is the spell out of a head that coordinates two VPs containing the verb and the object (if there is one). There is then one $v$ that takes this coordinated structure as its complement. The subject is introduced as the specifier of $v$, meaning that there is only one subject DP for the two coordinated predicates. This structure is illustrated in (6).

(6)

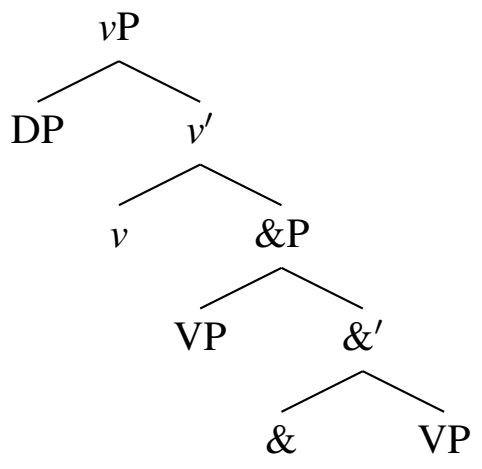

SS

In this structure, the SS marker is the coordinator (represented with \&) of two VPs. There is only one subject DP and it is introduced by $v$ above the height of coordination.

Keine argues that DS structures involve high coordination, at the $v \mathrm{P}$ level. The DS marker, under this account, spells out a head that coordinates two $v$ Ps, which each contain a distinct instance of an external argument. A DS structure is given in (7).

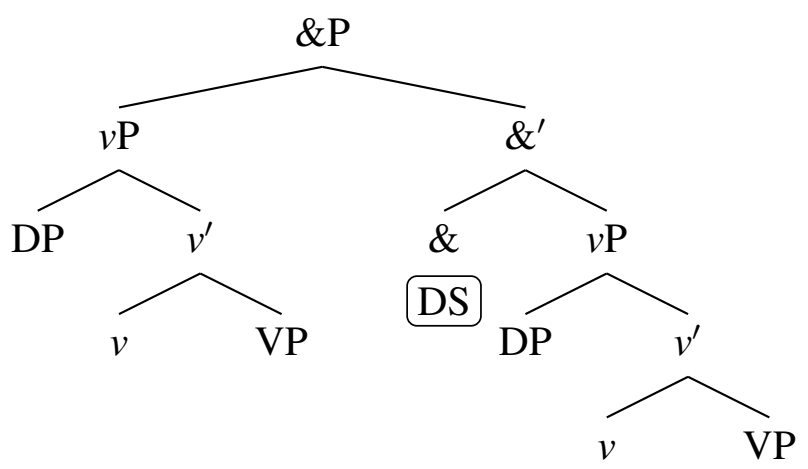

We see in this structure that the coordinator of the two $v$ Ps is the DS marker. Each $v \mathrm{P}$ contains a DP specifier, so there are two subjects in this construction.

As with Georgi's account, one important facet of Keine's analysis is that it predicts that SS clauses should only be able to have one instance of a subject DP since the subject is introduced above the height of coordination. Also like Georgi's account, this analysis predicts that 
SS structures should be smaller than DS structures. With these predictions in mind, I turn to a discussion of further data from Amahuaca, which pose a problem for the analyses outlined here.

4. Multiple overt subjects in Amahuaca same subject constructions. In Amahuaca SS constructions, an overt subject can appear in the reference clause, as in (8a), or in the marked clause, as in $(8 b){ }^{4}$

'While singing, the children dance.'
a. $\quad[$ vua $=$ hi $]=$ mun $\quad$ chirin $=$ hi $\quad$ vaku=vo $=\mathrm{ki}=\mathrm{nu}$ sing $=$ SS.SIM. $N O M=C$ dance $=I P F V$ child $=P L=3 . P R E S=D E C L$
b. $\quad[\mathbf{v a k u}=\mathbf{v o}$ vua $=$ hi $]=$ mun $\quad$ chirin $=\mathrm{hi}=\mathrm{ki}=\mathrm{nu}$ child $=\mathrm{PL}$ sing $=\mathrm{SS}$.SIM. $\mathrm{NOM}=\mathrm{C}$ dance $=\mathrm{IPFV}=3 . \mathrm{PRES}=\mathrm{DECL}$

The fact that a subject can appear in the marked clause is already potentially problematic for a control-based account like that of Georgi (2012). This is because the overt controller would be in the lower clause. In order for this to work, we would have to assume that backward control was an option (Polinsky \& Potsdam, 2002).

More problematic, however, than the variability of subject placement is the fact that an overt subject can appear in both clauses simultaneously, as demonstrated in (9). ${ }^{5}$

$$
\begin{aligned}
& {[(\mathbf{x a n o}=\mathbf{n}) \text { hatza vana }=\mathrm{xOn}]=\operatorname{mun} \quad(\mathbf{x a n o}=\mathbf{n}) \quad \text { jiriti vuna=hi }} \\
& \text { woman=ERG manioc plant=SS.SQ.ERG=C } \text { woman=ERG food look.for=IPFV } \\
& \text { jan=ki=nu } \\
& 3 \mathrm{SG}=3 . \mathrm{PRES}=\mathrm{DECL} \\
& \text { 'After planting manioc, the woman is looking for food.' }
\end{aligned}
$$

The sentence in (9) is immediately an issue for Keine's (2012; 2013) account based on coordination height. If SS constructions involve VP coordination, there should be only one external argument position and it should be above the height of the coordination. Expressing the external argument overtly twice, once in each conjunct, should not be possible. These data thus suggest that an account of SR based on coordination above or below the position of the subject is not an adequate account of the Amahuaca data. ${ }^{6}$

\footnotetext{
${ }^{4}$ Note that marked clauses are shown before the second position clitic $=m u n$, which is always preceded by exactly one constituent. While this is not the only possible position for marked clauses, I show them in this position to ensure that what is inside the square brackets of the marked clause is actually a constituent with the marked clause.

${ }^{5}$ Some speakers readily accept this type of example with two identical full DPs, while others find such examples to be degraded. Speakers who disprefer such constructions often comment that it sounds odd to repeat the noun, but they accept two pronominal arguments in the same configuration. This reaction indicates that these examples are perhaps not unlike similar English examples which seem slightly strange such as 'After the $\operatorname{woman}_{i}$ planted manioc, the $\operatorname{woman}_{i}$ looked for food.'

${ }^{6}$ Keine does admit the possibility that for some languages the height of SR coordination may actually be higher in the clause. Specifically, he argues that SS marking may indicate TP coordination and DS marking may indicate CP coordination in some languages. However, the language for which he proposes this solution is Kiowa, which exhibits non-canonical SR marking (also called unexpected SS/DS marking). McKenzie (2012) argues that non-canonical SR in Kiowa tracks topic situations rather than arguments. Thus, Keine argues that SS coordination in Kiowa involves only one topic situation, while DS coordination coordinates CPs, allowing for two distinct topic situations. Crucially, Amahuaca does not exhibit the type of non-canonical SR patterns that would be expected if it were topic situations
} 
The presence of two subjects in (9) is also problematic for the control-based account of Georgi (2012). Recall that Georgi assumes the movement theory of control. However, she argues that movement does not leave a copy. If we modify this assumption, we could potentially say that in (9), the two instances of the subject are two copies in a movement chain and that both are pronounced due to some special exception to normal chain resolution algorithms. Interestingly, though, when two subjects appear in an SS construction, they need not be formally identical. For example, in (10) the two subjects mismatch in case.

$$
\begin{aligned}
& {[(\mathbf{h i y a}=\mathbf{n}) \text { hatza vana }=\text { hax }]=\text { mun } \quad(\text { hiya=x }) \text { kaan=hi hun=ka=nu }} \\
& 1 \mathrm{SG}=\mathrm{ERG} \text { manioc plant=SS.SQ.NOM=C } 1 \mathrm{SG}=\mathrm{NOM} \text { walk=IPFV } 1 \mathrm{SG}=1 . \mathrm{PRES}=\mathrm{DECL} \\
& \text { 'After planting manioc, I am walking.' }
\end{aligned}
$$

In (10), the first person pronoun surfaces with ergative case in the transitive marked clause but with nominative case in the intransitive reference clause. In order to account for this under an analysis like Georgi's we would have to assume that different copies in the same movement chain can be assigned different case.

In (11), however, we see that mismatches between the two subjects in an SS construction in Amahuaca can go beyond case.

$$
\begin{aligned}
& {[\mathbf{j o n i}=\mathbf{x} \quad \text { vua }=\text { kin }]=\text { mun } \quad(\text { jato=n) hatza } \quad \text { vana }=\text { hi } \quad k a n=k i=n u} \\
& \text { man }=\text { NOM sing }=\text { SS.SIM.ERG }=C \text { C } P L=E R G \text { manioc } p l a n t=I P F V ~ 3 P L=3 . P R E S=D E C L \\
& \text { 'While the men are singing, they are planting manioc.' }
\end{aligned}
$$

The marked clause in (11) contains the DP jonix 'men'. The coreferential reference clause argument is a third person plural pronoun jaton. In order to maintain that this was a control structure with movement, we would have to allow one copy in a chain to be spelled out as a full NP and another to be spelled out as a pronoun.

Another mismatch between subjects in an SS construction is shown in (12).

$$
\begin{aligned}
& {[\text { hatza kiyoo=pan jova }=\text { xon }]=\text { mun } \quad \text { xano }=\text { n kuntii choka }=\mathrm{xO}=\mathrm{nu}} \\
& \text { manioc all=ERG cook }=\mathrm{SS} . \mathrm{SQ} . \mathrm{ERG}=\mathrm{C} \text { woman }=\mathrm{ERG} \text { pot wash }=3 . \mathrm{PST}=\mathrm{DECL} \\
& \text { 'After everyone cooked manioc, the woman washed the pot.' }
\end{aligned}
$$

What is interesting about (12) is that the mismatch here goes beyond the form of the DP. Here, there is not strict identity of reference between the subject of the marked clause and the reference clause. In fact, the subject of the marked clause is a quantified DP, a non-referring expression. As long as the subject of the reference clause xanon 'woman' is understood to be one of the individuals who cooked manioc, however, SS marking is licit. It is unclear how such a pattern could be derived via movement of a DP from the marked clause into the reference clause.

The data we have considered here, where an overt subject can appear in both clauses of an SS construction, are not in line with the prediction of the non-reference-tracking theories that SS constructions should contain only one subject DP. However, I will show in the next section that the other prediction of these theories - that there should be a size asymmetry between SS and DS clauses - is borne out in Amahuaca.

rather than arguments that were tracked by the language's SR system. 
5. The size of Amahuaca marked clauses. The accounts outlined in Section 3 both predict that SS marked clauses should be smaller than DS marked clauses. For Georgi (2012), SS clauses should be defective TPs while DS clauses should be full CPs. For Keine (2012, 2013), SS clauses should be VPs while DS clauses should be $v$ Ps. Such a size assymetry is not predicted by the direct reference-tracking accounts of Finer $(1984,1985)$ and Watanabe (2000), which take both DS and SS clauses to be full CPs differing only in the form of the complementizer.

Interestingly, in Amahuaca we do see evidence that SS clauses are indeed smaller than DS clauses. Amahuaca has a series of phonologically weak person markers which indicate the person and number features of the subject. In matrix clauses, these morphemes are obligatory for local persons and optionally double full NPs for third persons. In DS clauses we see the same pattern of obligatoriness as in matrix clauses. This is demonstrated with the first person subject of the marked clause in (13).

$$
\begin{aligned}
& {[* \text { (hun) nokoo }=\text { kun }]=\text { mun jan hoxa }=\mathrm{xo}=\mathrm{nu}} \\
& 1 \mathrm{SG} \quad \text { arrive }=\mathrm{DS} . \mathrm{SQ}=\mathrm{C} \quad 3 \mathrm{SG} \text { sleep }=3 . \mathrm{PST}=\mathrm{DECL} \\
& \text { 'After I arrived, he slept.' }
\end{aligned}
$$

In (13), the first person singular marker hun is obligatory in the DS marked clause. This contrasts with the pattern found in SS clauses where these markers are ungrammatical, as seen in (14).

$$
\begin{aligned}
& {[(* \text { hun }) \text { nokoo }=\text { hax }]=\text { mun hun hoxa }=\mathrm{ku}=\mathrm{nu}} \\
& 1 \mathrm{SG} \text { arrive=SS.SQ.NOM=C } 1 \mathrm{SG} \text { sleep }=1 . \mathrm{PST}=\mathrm{DECL} \\
& \text { 'After arriving, I slept.' }
\end{aligned}
$$

We see in (14) that it is impossible for the first person marker to surface in the SS marked clause, even though it is required in the matrix clause. This is in contrast with the distribution of full DP subjects, which can appear in both the marked and reference clause in SS constructions, as demonstrated in Section 4.

The distribution of person markers in SS and DS clauses suggests that DS clauses are larger. Only DS clauses are of a sufficient size to host person marking. This is in line with predictions of the non-reference-tracking theories discussed in Section 3. Both accounts predict that DS clauses should be larger than their SS counterparts. The size asymmetry seen in the Amahuaca data are not predicted by direct reference-tracking theories. There seems to be a conflict between the two pieces of Amahuaca evidence we have considered here. The distribution of overt DP subjects is not compatible with non-reference-tracking theories, but the size asymmetry is not predicted by reference-tracking theories. Thus, while we cannot adopt a non-reference-tracking theory for the Amahuaca data, traditional reference-tracking theories will need to be adapted as well in order to account for the data.

6. Conclusion. I have demonstrated that Amahuaca SS constructions simultaneously allow two instances of an overt DP subject - one in the marked clause and one in the reference clause. This pattern does not align with the predictions of Georgi's (2012) control-based account of SR or Keine's $(2012 ; 2013)$ coordination-based account of SR, since both of these accounts predict that there should be only one subject DP in an SS construction. However, the Amahuaca data also do not match the predictions of traditional direct-reference-tracking accounts, but in a different way. Evidence from the distribution of person markers in marked clauses suggests 
that SS marked clauses are structurally smaller than DS marked clauses. This asymmetry is in line with the predictions of non-reference-tracking accounts but not the more traditional accounts of SR based on direct reference tracking. Therefore, the Amahuaca data provide evidence that, while we cannot adopt the non-reference-tracking theories of Georgi (2012) and Keine $(2012,2013)$, neither do the direct reference-tracking accounts of Finer $(1984,1985)$ and Watanabe (2000) paint the full picture. Instead, we need an account that does not derive the SS versus DS contrast from the number of DPs in the structure but that still allows for SS derivations to contain less structure than their DS counterparts.

\section{References}

Boeckx, Cedric, Norbert Hornstein \& Jairo Nunes. 2010. Control as movement, Cambridge Studies in Linguistics, vol. 126. New York: Cambridge University Press.

Finer, Daniel L. 1984. The formal grammar of switch-reference. University of Massachusetts, Amherst dissertation.

Finer, Daniel L. 1985. The syntax of switch-reference. Linguistic Inquiry 16.35-55.

Georgi, Doreen. 2012. Switch-reference by movement. In Philipp Weisser (ed.), Perspectives on switch-reference: Local modeling and empirical distribution, 1-40. Leipzig: Institute für Linguistik, Universität Leipzig.

van Gijn, Rik. 2016. Switch reference in Western South America. In Rik van Gijn \& Jeremy Hammond (eds.), Switch reference 2.0, 153-206. Amsterdam: John Benjamins.

Jacobsen, William H. 1967. Switch-reference in Hokan-Coahuiltecan. In Dell H. Hymes \& William E. Bittle (eds.), Studies in South-Western ethnolinguistics, 238-263. The Hague: Mouton.

Keine, Stefan. 2012. Switch-reference as coordination. In Philipp Weisser (ed.), Perspectives on switch-reference: Local modeling and empirical distribution, 107-164. Leipzig: Institute für Linguistik, Universität Leipzig.

Keine, Stefan. 2013. Deconstructing switch-reference. Natural Language \& Linguistic Theory 31.767-826.

McKenzie, Andrew. 2012. The role of contextual restriction in reference-tracking. University of Massachusetts, Amherst dissertation.

Munro, Pamela (ed.). 1979. Studies of switch-reference, UCLA Papers in Syntax, vol. 8. Los Angeles: University of California.

Polinsky, Maria \& Eric Potsdam. 2002. Backward control. Linguistic Inquiry 33.245-282.

Simons, Gary F. \& Charles D. Fennig (eds.). 2017. Ethnologue: Languages of the world. Twentieth edn. Dallas: SIL International. Online version: http://www.ethnologue.com.

Sparing-Chávez, Margarethe. 1998. Interclausal reference in Amahuaca. In Desmond C. Derbyshire \& Geoffrey K. Pullum (eds.), Handbook of Amazonian languages, vol. 4, 443485. Berlin: Mouton de Gruyter.

Sparing-Chávez, Margarethe. 2012. Aspects of Amahuaca grammar: An endangered language of the Amazon basin. Dallas: SIL International.

Valenzuela, Pilar. 2003. Transitivity in Shipibo-Konibo grammar. University of Oregon dissertation.

Watanabe, Akira. 2000. Feature copying and binding: Evidence from complementizer agreement and switch reference. Syntax 3.159-181. 